\title{
Pseudo occlusion-based 3D trajectory visualization for indoor AR navigation services
}

\author{
Xiangling Peng a,*, Hayato Aoyagi ${ }^{\mathrm{b}}$, Masatoshi Arikawa ${ }^{\mathrm{a}}$, Sato Ryo ${ }^{\mathrm{a}}$, Akinori Takahashi ${ }^{\mathrm{a}}$ \\ ${ }^{a}$ Akita University, Graduate School of Engineering Science, Human-Centered Computing Course, m8020551@ s.akita-u.ac.jp \\ ${ }^{b}$ Akita University, Faculty of Engineering Science, Human-Centered Computing Course \\ * Corresponding author
}

Keywords: pseudo occlusion, augmented reality, 3D (three-dimensional) trajectories, indoor navigation, geofences

\begin{abstract}
:
Nowadays, with the development of AR technology, it is popular to use AR trajectory (Figure 1) instead of 2D (twodimensional) trajectory to guide us in navigation applications (Hua and Wijkmark, 2019). Differed from conventional outdoor navigation services, indoor navigation services require more in the location accuracy and trajectory legibility performance. Firstly, using GPS to locate the users' indoor location is not feasible. Generally, the location accuracy of GPS only controls the error in about three or five meters which is not enough for the precise requirement of indoor navigation applications. Motion sensors are getting common to use instead of GPS to achieve high accuracy in positioning for indoor navigation. Also, the indoor GPS signal is useless, and could make the positioning result worse. Furthermore, visualizing trajectories of the indoor navigation needs more computing performance compared with the cases of outdoor navigation because of occlusion processing in 3D complex indoor spaces. For instance, some complex building structures needs high computing performance of visualizing trajectories of users consistently solving occlusion problems inside precisely. When a user would turn a corner or meet obstacles, the trajectory in other side should be invisible. Using occlusion for the trajectory display to be more coordinate with the real-world logic is practical. However, building and computing the present real occlusion based on 3D spatial data costs high and is difficult to realize versatilely. Therefore, it is important to find a way to perform the trajectory well and simple to realize. This research proposed a new method called pseudo occlusion which costs low and is simple to realize visualization of the trajectory in 3D spaces by using only point objects, that is a circular-type geofence, and any other high-dimensional objects such as building element objects including walls and exhibited objects and so on in 3D spaces. Thus, our proposed method does not need to build 3D spatial data and perform a satisfying occlusion result of trajectory.
\end{abstract}

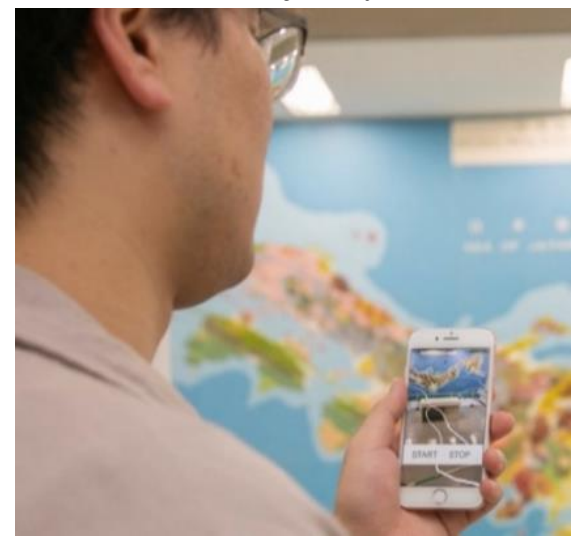

Figure 1. An example of 3D CG trajectory objects as a white line shows in an AR application on an Apple Inc.'s iPhone (ARKit, 2021).

Compared with presenting the real occlusion, our proposed pseudo occlusion has more advantages on indoor navigation practical use. Firstly, it is difficult to build real 3D spatial objects. The measurement data of the building is not only hard to obtain, but also takes a lot of time to build a 3D model. The pseudo occlusion does not need to build 3D models or objects and still achieve the wanted occlusion result. Secondly, the calculation of making the real occlusion costs high for a smartphone and would degrade the quality of service. The pseudo occlusion calculation occupies much lesser storage than real occlusion building. Furthermore, the real 3D volume spatial data are difficult to be created by end-users. It constrains the application fields in limited places because only professional spatial data developers can create them. But the pseudo occlusion only need simple point data in 3D spaces which can be created by ordinary users as SNS.

To realize the pseudo occlusion, there are two methods, that is, i) room (check-in/out) geofence and ii) visible distance. Firstly, the geofence is a location-based service when you would enter in a defined area, events would be triggered and take some actions (Figure 2). We set different space categories such as corridors, rooms, stairs and so on (Gautrand, 2014). Gateways of each scene we would set a center point and a radius of it for a room geofence. It is one-dimensional 
data which can be created at a low cost and processed with light computing. When we step in a different scene, the room geofence is triggered and trajectory visualization are changed correspondingly so that the pseudo occlusion can be achieved. Secondly, sometimes the room shape is not regular, and some part of the room is invisible, although it is still in the room defined by the room geofence, the trajectory of this hidden part of the room should not be seen. Setting a limit for trajectory visible distance is also needed for visibility control of trajectory. The combination of the room geofence and the trajectory visible distance settings enable a satisfying trajectory guide on indoor navigation (Figure 3 ).
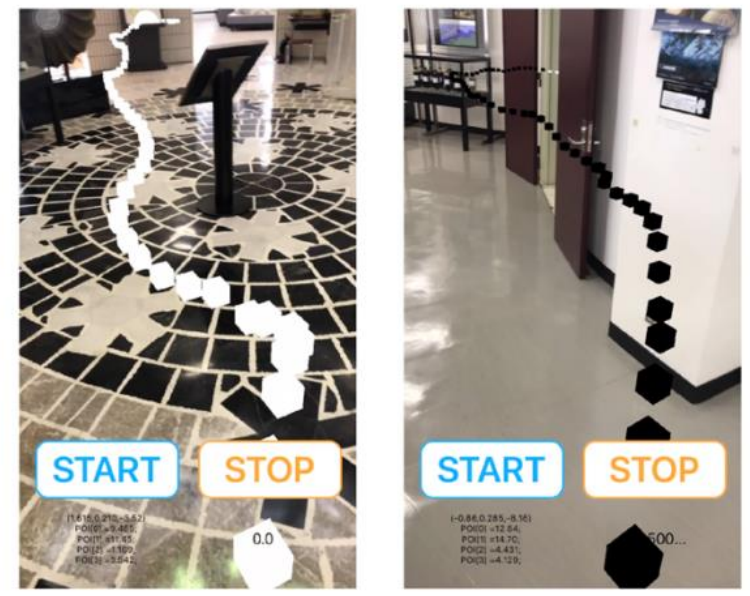

Figure 2. The experiments of the room geofence to visualize trajectories in different colors due to different space categories such as a corridor (left) and an exhibition room (right).
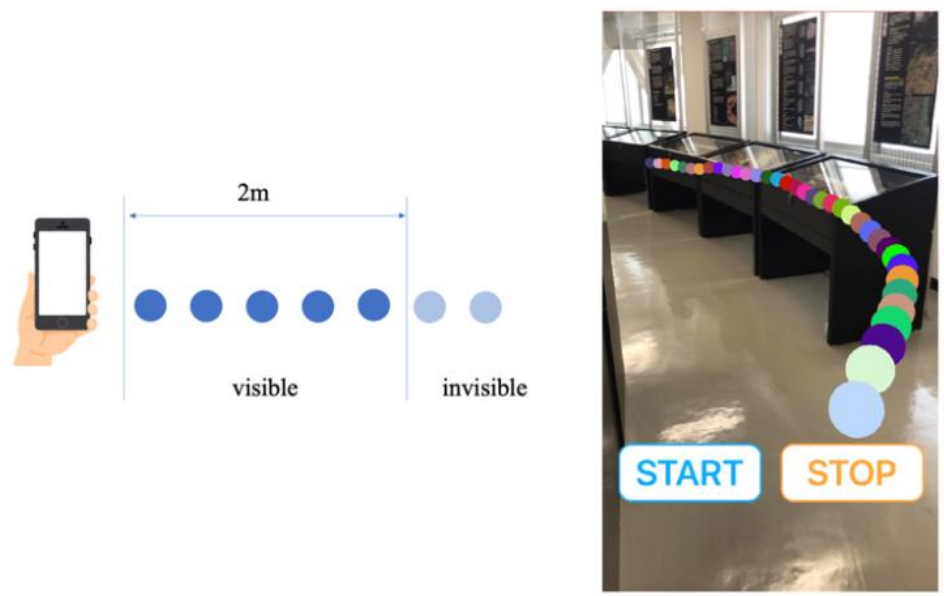

Figure 3. Trajectory CG objects could be visible only if the distance between a smartphone (a user) and each of the trajectory objects is less than two meters.

The experiments we have done have proved that it could work at a real museum (Sparacino, 2002), that is, Mining Museum of Akita University. We chose two meters for the setting of trajectory visible distance limit. When we walked through a corridor and entered in an exhibition room, the room geofence would be triggered, then the trajectory CG objects were changed in the color appropriately and quit showing the previous trajectory passing through the corridor.

This method of AR trajectory visualization could keep the users feeling natural without building a three-dimension indoor model and with a local coordinate system, but not a global one. It could decrease the calculation burden for smartphones and easier to realize. We found that there are still some works to improve in the method. The detection of occlusion largely relies on the accuracy of geofence settings, when some irregular shapes of the room structures are not suitable for the room geofence settings, the precision of determination would be impacted.

\section{References}

Hua, A. and Wijkmark, R., 2019, Evaluation of Mobile Augmented Reality for Indoor Navigation, Examensarbete Inom Datateknik, Grundniva, 15 HP, Stockholm, Sverige.

ARKit, 2021, Apple Inc., https://developer.apple.com/augmented-reality/arkit/.

Gautrand, M., et al., 2014, Eds., Museum Architecture and Interior Design, Design Media Pub Ltd.

Sparacino, F., 2002, The Museum Wearable: real-time sensor-driven understanding of visitors' interests for personalized visually-augmented museum experiences, Proceedings of Museums and the Web, Boston, US. 